\title{
Integration of Artificial Intelligence into Dempster Shafer theory: A review on decision making in condition monitoring
}

\author{
M.F.Rosli ${ }^{1, a^{*}}, \mathrm{~L} \mathrm{M} \mathrm{Hee^{2,b }}$ and M. Salman Leong ${ }^{3, c}$ \\ ${ }^{1,2}$ RAZAK School of Engineering \& Advanced Technology, Universiti Teknologi Malaysia, Malaysia \\ ${ }^{3,4}$ Faculty of Mechanical Engineering, Universiti Teknologi Malaysia, Malaysia \\ aroslifirdaus@yahoo.com, ${ }^{\mathrm{b}} \mathrm{mhlim@ic.utm.my,}{ }^{\mathrm{c}}$ salman@ic.utm.my
}

Keywords: Dempster-Shafer, Support Vector Machine, Basic probability assignments

\begin{abstract}
Machines are the heart of most industries. By ensuring the health of machines, one could easily increase the company revenue and eliminates any safety threat related to machinery catastrophic failures. In condition monitoring $(\mathrm{CM})$, questions often arise during decision making time whether the machine is still safe to run or not? Traditional CM approach depends heavily on human interpretation of results whereby decision is made solely based on the individual experience and knowledge about the machines. The advent of artificial intelligence (AI) and automated ways for decision making in CM provides a more objective and unbiased approach for CM industry and has become a topic of interest in the recent years. This paper reviews the techniques used for automated decision making in CM with emphasis given on Dempster-Shafer (D-S) evident theory and other basic probability assignment (BPA) techniques such as support vector machine (SVM) and etc.
\end{abstract}

\section{Introduction}

There has been a significant increase in the number of $\mathrm{CM}$ tools and techniques since the acceptance of CM practices in the field. Tools such as vibration analysers, infrared thermography camera, tribology analysis and so forth have flourished on the market over the years. Beside, many advanced technique had also been incorporated into these tools to make the results more reliable and accurate. Traditionally, CM of machines was done based on the monitoring of individual parameters such as vibration, pressure and etc. However, it is believed that a better approach for $\mathrm{CM}$ is to perform data fusion and correlation analysis on all available parameters or sensory data which could bring more insight into the health of the machine. Techniques such as Neural Networks $(\mathrm{NN})$, fuzzy logic, expert system, Bayesian, model based reasoning and D-S could be used to achieve this purpose and to help in decision making process in CM. In this paper, D-S technique will be discussed as the technique currently appeared to be at the forefront of research for machinery diagnosis and decision making process.

\section{D-S Theory}

On 1976, Shafer established the Dempster theory which is proposed as an alternative to Bayesian inference theory. The capability of D-S to model imprecisions and randomness makes it a more superior theory than Bayesian approaches for modelling of uncertainty profiles. A frame of discernment, $\Theta$ which contained every possible hypothesis is introduced, $\Theta=\{F i \mid i=1,2,3 \ldots\}$ Basic probability assignment (BPA) denotes by $m$ is defined as a set of power set $2^{\ominus}$ such that BPA of an empty set denotes by $\phi$ is 0 and the summation of hypothesis is 1 .

$$
\begin{array}{cc}
\mathrm{m}: 2^{\Theta} \rightarrow[0,1] \quad \mathrm{m}(\phi)=0 \quad \text { and } & \sum_{X \subseteq \theta} m(X)=1 \\
\mathrm{~m}(\mathrm{C})=\left\{\begin{array}{lr}
0 & \text { if } X \cap Y=\phi \\
\frac{\sum_{x \cap Y=C, \text { For all } X, Y \subseteq \theta} m_{i}(X) \times m_{i^{\prime}}(Y)}{1-\sum_{x \cap Y=\phi, \text { For all } X, Y \subseteq \theta} m_{i}(X) \times m_{i^{\prime}}(Y)} & \text { if } X \cap Y \neq \phi
\end{array}\right.
\end{array}
$$


$\operatorname{Bel}(X)=\sum_{y \subseteq x} \mathrm{~m}(\mathrm{Y})$

$P l(X)=\sum_{\mathrm{y} \cap \mathrm{xA} \neq \Phi} \mathrm{m}(\mathrm{Y})$
For any $\mathrm{X} \subseteq \Phi$

For any $\mathrm{X} \subseteq \Phi$ and all $Y \subseteq \Phi$

Problem arises from this combination rules are such as evidence sufficiency $\mu$, evidence importance and conflicting evidences which were solved by introducing the fuzzy membership function, important index, $v$ and conflict factor, $\mathrm{k}$ as proposed by other researches [1]. Data combination may be performed at 3 different levels namely data level, feature level and decision level. Data of different sensors, inputs or different sensor types and parameters can be analysed using D-S combination rules to achieve a more accurate and reliable results for $\mathrm{CM}$ of machine.

\section{BPA}

In D-S theory, BPA can be determined using either the expert opinion, flat BPAs, occurrence frequency, Gaussian distribution modelling and etc. Nevertheless in CM application, BPA is always determined by expert opinion. The advent of AI enabled researchers to use these techniques to minimize bias during BPA assignments problems. For BPA based on fuzzy membership function and diagnostic decision rules are given as [2]. According to Guang and $\mathrm{Wu}$, correlation coefficient selection is inconsistent and subjective. Thus, fuzzy membership function, $\mu_{\mathrm{i}}$ have been represented as per figure 1. It was showed that with the fusion of multi-faults characteristics, the credibility of diagnostic results increased and its uncertainty decreased evidently, which demonstrates the feasibility fuzzy logic and D-S evidence theory for machinery fault diagnosis.

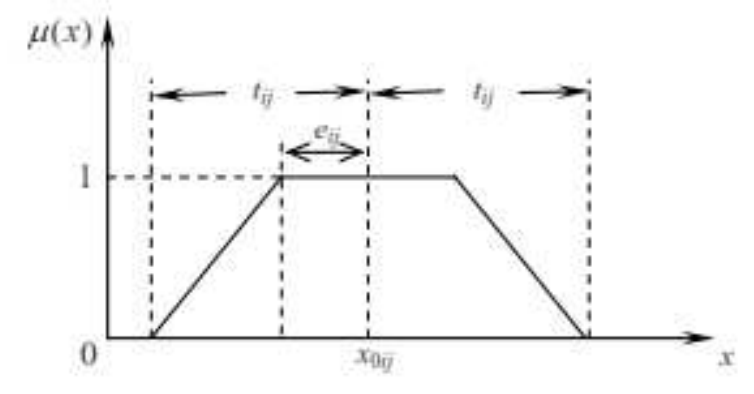

Figure 1: Fuzzy membership function of eigenvalue [2]

\section{NN in D-S application}

The basic of NN was introduced in 1943 which consists of 3 different layers of input, hidden and output layers. Common NN techniques that were widely used in D-S analysis are back propagation neural network (BPNN) and artificial neural network (ANN). The structure of BPNN is shown in figure 2.

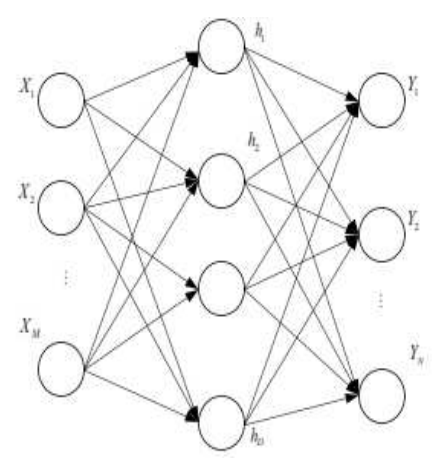

Figure 2: BPNN structure [3]

Yibo et al. in [3] have demonstrated the effectiveness of data fusion between vibration and oil analysis using D-S with BPA from BPNN output and for fault diagnosis of gear box. While Khazaee et al. in [4] have reported an increase of accuracy in machinery fault diagnosis via ANN- 
DS method where ANN output were used as BPA. Ai and Cheng [5] has introduced Particle swarm algorithm (PSO) to improve the convergence speed of BPNN. This method improves BPNN generalization ability and cause the output error is to be small which in turn verified the effectiveness of using improved BPNN output as BPA for results fusion.

\section{SVM in D-S application}

Recently, SVM were used extensively in many researches for machinery fault diagnosis such as in rolling element bearing (REB) [6], reciprocating compressor valve [7], induction motors [8] and gears [9]. For non-linear case of SVM the equation can be expressed as:

$w(\phi)+b=0$

$\mathrm{f}(\mathrm{x})=\operatorname{sgn}(\mathrm{w}(\phi)+\mathrm{b})=\operatorname{sgn}\left\{\sum_{i=1}^{l} a_{i}^{*} y_{i} K\left(x_{i}, x\right)+b^{*}\right\}$

Where $K\left(x_{i}, x\right)$ is the function of Kernel (such as Gaussian, polynomial or linear). Then, the output of SVM were used as D-S's BPA [10]. Besides machinery fault diagnosis, SVM-DS method were also used in character recognition research according to Guo and Zhang [11]. Another evolution of BPA selection is to use Least squares SVM (LSSVM) which Lu et al. have compared the LSSVM with Back Propagation Neural Network (BPNN) and Radial Based Function Neural Network (RBF-NN) to compare the accuracy of LSSVM and D-S [12]. It was reported that the advantages of SVM technique as compare to other machine learning techniques such as can be used for small samples, sample's non-linearity, over fitting, local minimum value and also has excellent generalization ability. Recently, there have been many attempts to introduce wavelet kernel into SVM analysis due to its ability to extract characteristics features of the time series. This introduction of wavelet SVM (w-SVM) can further increase the ability of SVM in handling different categories of bearing faults, stock market, medical field and induction machinery faults. Detailed discussion of w-SVM could be found in [13].

\section{Conclusion}

D-S offers a potentially good solution for decision making of CM and machinery fault diagnosis. It gives quantitative value on the confident level of the results to be used during the decision making stage in CM. An important stage of D-S analysis is in BPA assignment process. By incorporating AI techniques such as ANN, fuzzy logic and other expert system has many disadvantages such as poor generalization when the number of fault samples is low, low convergence rate and obvious over fitting. SVM is known to be a better choice as these issues pertaining to BPA assignment could be eliminated or solved in a straight forward manner.

\section{Acknowledgement}

This work is supported by the UTM Institute of Noise and Vibration and Research University Grant of UTM (Q.K130000.2540.06H51 and Q.J130000.2524.05H01) financed by the Ministry of Education, Malaysia. 


\section{References}

[1] X. Fan and M. J. Zuo, "Fault diagnosis of machines based on D-S evidence theory. Part 1: D-S evidence theory and its improvement," Pattern Recognit. Lett., vol. 27, no. 5, pp. 366376, Apr. 2006.

[2] G. Yang and X. Wu, "Synthesized Fault Diagnosis Method Based on Fuzzy Logic and D-S Evidence Theory *," pp. 1024-1031.

[3] C. Yibo, X. Xiaopeng, L. Yan, and D. Tianhuai, "Fault Diagnosis of Gear Using Oil Monitoring Samples and Vibration Data," pp. 934-939.

[4] M. Khazaee, H. Ahmadi, M. Omid, and a. Moosavian, "Classifier fusion of vibration and acoustic signals for fault diagnosis and classification of planetary gears based on DempsterShafer evidence theory," Proc. Inst. Mech. Eng. Part E J. Process Mech. Eng., vol. 228, no. 1, pp. 21-32, Jan. 2013.

[5] L. Ai and J. Cheng, "FAULT DIAGNOSIS OF ROLLING BEARING BASED ON MULTISENSOR INFORMATION FUSION,” pp. 1043-1045.

[6] D. Fernández-Francos, D. Martínez-Rego, O. Fontenla-Romero, and A. Alonso-Betanzos, "Automatic bearing fault diagnosis based on one-class v-SVM," Comput. Ind. Eng., vol. 64, no. 1, pp. 357-365, Jan. 2013.

[7] H. Cui, L. Zhang, R. Kang, and X. Lan, "Research on fault diagnosis for reciprocating compressor valve using information entropy and SVM method," J. Loss Prev. Process Ind., vol. 22, no. 6, pp. 864-867, Nov. 2009.

[8] L. M. R. Baccarini, V. V. Rocha e Silva, B. R. de Menezes, and W. M. Caminhas, "SVM practical industrial application for mechanical faults diagnostic," Expert Syst. Appl., vol. 38, no. 6, pp. 6980-6984, Jun. 2011.

[9] D. J. Bordoloi and R. Tiwari, "Support vector machine based optimization of multi-fault classification of gears with evolutionary algorithms from time-frequency vibration data," Measurement, vol. 55, pp. 1-14, Sep. 2014.

[10] L. Zhang and Y. Dong, "Research on Diagnosis of AC Engine Wear Fault Based on Support Vector Machine and Information Fusion," J. Comput., vol. 7, no. 9, pp. 2292-2297, Sep. 2012.

[11] X. C. Guo and S. Q. Zhang, "Mine Loadometer License Plate Recognition Based on MFFDS," Adv. Mater. Res., vol. 712-715, pp. 2341-2344, Jun. 2013.

[12] F. Lu, T. Bin Zhu, and Y. Q. Lv, "Data-Driven Based Gas Path Fault Diagnosis for TurboShaft Engine," Appl. Mech. Mater., vol. 249-250, pp. 400-404, Dec. 2012.

[13] Z. Liu, H. Cao, X. Chen, Z. He, and Z. Shen, "Multi-fault classification based on wavelet SVM with PSO algorithm to analyze vibration signals from rolling element bearings," Neurocomputing, vol. 99, pp. 399-410, Jan. 2013. 